\title{
The additional value of first pass myocardial perfusion imaging during peak dose of dobutamine stress cardiac MRI for the detection of myocardial ischemia
}

\author{
Daniel D. Lubbers · Caroline H. C. Janssen • \\ Dirkjan Kuijpers · Paul R. M. van Dijkman • \\ Jelle Overbosch · Tineke P. Willems - Matthijs Oudkerk
}

Received: 26 October 2006/ Accepted: 23 December 2006/Published online: 14 June 2007

(C) Springer Science+Business Media B.V. 2007

\begin{abstract}
Purpose of this study was to assess the additional value of first pass myocardial perfusion imaging during peak dose of dobutamine stress Cardiac-MR (CMR). Dobutamine Stress CMR was performed in 115 patients with an inconclusive diagnosis of myocardial ischemia on a $1.5 \mathrm{~T}$ system (Magnetom Avanto, Siemens Medical Systems). Three short-axis cine and grid series were acquired during rest and at increasing doses of dobutamine (maximum $40 \mu \mathrm{g} / \mathrm{kg} / \mathrm{min}$ ). On peak dose dobutamine followed immediately by a first pass myocardial perfusion imaging sequence. Images were graded according to the sixteen-segment model, on a four point scale. Ninety-seven patients showed no New (Induced) Wall Motion Abnormalities (NWMA). Perfusion
\end{abstract}

D. D. Lubbers $(\varangle) \cdot$ C. H. C. Janssen ·

D. Kuijpers · J. Overbosch · T. P. Willems ·

M. Oudkerk

Department of Radiology, University Medical Center Groningen, University of Groningen, Hanzeplein 1,

Groningen $9700 \mathrm{RB}$, The Netherlands

e-mail: d.d.lubbers@rad.umcg.nl

\section{Kuijpers}

Department of Radiology, Bronovo Hospital,

Bronovolaan 1, P.O.Box 96900, The Hague 2597 AX, The Netherlands

\section{P. R. M. van Dijkman}

Department of Cardiology, Bronovo Hospital,

Bronovolaan 1, P.O.Box 96900, The Hague 2597 AX, The Netherlands imaging showed absence of perfusion deficits in 67 of these patients (69\%). Perfusion deficits attributable to known previous myocardial infarction were found in 30 patients $(31 \%)$. Eighteen patients had NWMA, indicative for myocardial ischemia, of which 14 (78\%) could be confirmed by a corresponding perfusion deficit. Four patients $(22 \%)$ with NWMA did not have perfusion deficits. In these four patients NWMA were caused by a Left Bundle Branch Block (LBBB). They were free from cardiac events during the follow-up period (median 13.5 months; range 6-20). Addition of first-pass myocardial perfusion imaging during peak-dose dobutamine stress CMR can help to decide whether a NWMA is caused by myocardial ischemia or is due to an (inducible) LBBB, hereby preventing a false positive wall motion interpretation.

Keywords Heart · Ischemia · MRI .

Myocardium $\cdot$ Stress

\section{Introduction}

Dobutamine stress Cardiac MRI (CMR) is used to detect myocardial ischemia of the left ventricle by means of wall motion analysis during the infusion of high-dose dobutamine [1-4]. Previous studies have reported a broad range of sensitivity (83-91\%) and specificity (80-86\%) of dobuta- 
mine stress CMR for the detection of myocardial ischemia $[1-3,5]$. It has been proven to be more accurate than dobutamine stress echocardiography [2]. The addition of myocardial tagging even further increased the sensitivity (96\%) of dobutamine stress CMR [4]. False positive dobutamine stress CMR's were described for left bundle branch block (LBBB) or an incidental low interpretability [4]. Means to overcome for these falsepositive dobutamine stress CMR's could increase specificity even further.

Assessment of myocardial perfusion is used to provide information on the hemodynamic significance of a coronary artery stenosis. Segments with New Wall Motion Abnormalities (NWMA) detected with dobutamine stress CMR should also show perfusion deficits, since perfusion abnormalities precede wall motion abnormalities in the ischemic cascade [6].

Normal stress perfusion SPECT-results predict a less than $1 \%$ annual risk of cardiac death or myocardial infarction, thereby yielding a high negative predictive value [7-10]. High negative predictive values are also reported for MR perfusion imaging combined with MRI cineangiography $(100 \%)$ in a small study of 15 patients [11].

The addition of a perfusion sequence on peakdose dobutamine may further enhance the interpretation of dobutamine stress CMR, by ruling out false positive findings, through the combined strength of both methods.

The purpose of this study is to assess whether the addition of perfusion imaging to dobutamine stress CMR at peak-dose dobutamine reduces the number of false-positive dobutamine stress CMR examinations.

\section{Materials and methods}

\section{Patient population}

Between September 2004 and April 2006, 124 consecutive patients were referred from the department of Cardiology for a dobutamine stress CMR. The study was approved by the local ethical committee. Informed consent was obtained prior to the study, after the nature of the procedure had been explained. All patients had chest pain and an inconclusive diagnosis of coronary artery disease by means of history, ECG recordings at rest and, if performed, during a bicycle exercise test. Patients with an acute coronary syndrome, atrial fibrillation, severe arterial hypertension (>220/120), CMR-incompatible metallic implants or known claustrophobia were not eligible.

\section{Protocol for dobutamine stress CMR} with myocardial perfusion on peak-dose dobutamine

To ensure cardiac response to dobutamine, all anti-anginal medication was stopped 4 days before the dobutamine stress CMR examination. After the patient was positioned on the scanning table, intravenous access was established via an anticubital vein. ECG monitoring leads, a phasedarray surface coil covering the heart, and a brachial blood pressure cuff were applied. A single lead ECG was continuously monitored on the MRI-console. Systolic and diastolic blood pressures were recorded using an automatic device (Welch-Allyn, Emro-medical) at baseline and every $3 \mathrm{~min}$ throughout the procedure. Blood pressure and heart rate were recorded. The imaging methodology of dobutamine stress CMR has been described in detail previously [4]. Dobutamine stress CMR was performed on a $1.5 \mathrm{~T}$ system (Magnetom Avanto, Siemens Medical Systems, Erlangen, Germany). Three short-axis cine breath-hold CMR images of the left ventricle, with and without myocardial tagging, were acquired at rest and during incremental dosage of dobutamine up to $40 \mu \mathrm{g} / \mathrm{kg} / \mathrm{min}$. An ECG-triggered segmental gradient-echo pulse sequence was used: TrueFisp: TR 57.64, ms; TE, $1.1 \mathrm{~ms} ; \alpha, 59^{\circ}$; FOV, $284 \times 350 \mathrm{~mm}$; slice-thickness $6 \mathrm{~mm}$; and matrix $125 \times 192$; iPAT 2 . Tagging was performed with a standard FLASH grid-sequence: TR, $46 \mathrm{~ms}$; TE, $3.8 \mathrm{~ms} ; \alpha, 14^{\circ}$; FOV , $284 \times 350 \mathrm{~mm}$; slice-thickness $6 \mathrm{~mm}$ and matrix $141 \times 256$. The basal plane was taken $1.5 \mathrm{~cm}$ below the mitral valves. The midventricular and apical short-axis views were divided equally over the remaining part of the left ventricle. When a wall motion abnormality 
(WMA) was detected at baseline, infusion was started at $5 \mu \mathrm{g} / \mathrm{kg} / \mathrm{min}$, after which the dose of dobutamine was increased to $10,20,30$ and $40 \mu \mathrm{g} / \mathrm{kg} / \mathrm{min}$. Starting dose of dobutamine was $10 \mu \mathrm{g} / \mathrm{kg} / \mathrm{min}$ when no WMA was detected at baseline. Imaging started 6 min after each dose increase and required 3 min per dose increase.

Termination criteria for dobutamine stress CMR were the development of new wall motion abnormalities (NWMA) or worsening WMA, a fall of systolic blood pressure of more than $40 \mathrm{mmHg}$, marked hypertension above 240/ $120 \mathrm{mmHg}$, severe chest pain, ventricular arrhythmias and intolerable side effects. NWMA are indicative of myocardial ischemia.

On peak-dose dobutamine a bolus injection of $0.1 \mathrm{mmol} / \mathrm{kg}$ gadolinium-DTPA $\quad\left(\right.$ Omniscan ${ }^{\circledR}$ ) was given and a perfusion sequence was started: TrueFisp: TR, 150.5 or $163.1 \mathrm{~ms}$; TE $1.03 \mathrm{~ms}$; TI $100 / 103 \mathrm{~ms} ; \alpha 45 / 50^{\circ}$; FOV $300 \times 300$; slice-thickness $6 \mathrm{~mm}$; matrix $76 \times 128$; iPAT 2 . The same three slices as the cine and tagging series were acquired.

During the examination a radiologist present in the MR suite to monitor the condition of the patient and to visually evaluate the images. When NWMA's with a corresponding perfusion deficit were seen, a coronary angiography (CAG) was performed within 3 weeks. Patients with NWMA's and a normal first pass perfusion on peak stress entered follow-up.

\section{Image analysis}

Wall motion was scored on six segments of the basal plane, six on the midventricular plane and four on the apical plane. Segmental wall motion was qualitatively graded as $1=$ normal or hyperkinesis; 2 = hypokinesis; 3 = akinesis; and, 4 =dyskinesis. Myocardial ischemia was defined as a new (induced) or worsening WMA in at least two segments at consecutive planes of the left ventricle during infusion of dobutamine. Analysis was performed using both cine and tagging images as described in detail previously [4].

Wall Motion Score Index (WMSI) was derived as the mean score of all segments of all short-axis images. WMSI data from the combined analysis of the cine and tagging images were determined from baseline and peak stress images.

The first pass perfusion images on peak-dose dobutamine were visually analyzed by an experienced radiologist and cardiologist in a consensus reading after the wall motion analysis by the same physicians. A perfusion abnormality, corresponding to the coronary artery distribution areas, in at least two segments at consecutive short-axis slices or one segment of the most apical slice of the left ventricle was defined as myocardial ischemia.

Follow-up

Follow-up data were obtained in September 2006. The present status of the patient was determined by review of the hospital records or contacting the patient's general physician. The date of the last review was used to calculate follow-up time.

Evaluated end points were non-fatal myocardial infarction (angina of $>30$ min duration and either $2 \mathrm{~mm}$ ST segment elevation in two consecutive ECG leads or a rise in creatine kinase level and its myoglobine fraction two times the upper limit of normal), cardiac death (death in the presence of acute myocardial infarction, significant cardiac arrhythmias or refractory congestive heart failure) and coronary revascularization.

\section{Results}

Patient population

From the 124 consecutively included patients, in nine patients the examination could not be completed due to: intolerable side effects (nausea, vomiting) in four patients, claustrophobia in three patients. Two patients were excluded due to insufficient image quality, one of whom had an irregular rhythm with triggering difficulties, and the other patient was unable to sustain breath holds.

Therefore, 115 patients all with good image quality were analysed (93\%) with a mean age $61 \pm 11$ years, 20 women (30\%). Demographic and hemodynamic data are listed in Table 1. 
Table 1 Demographic and hemodynamic data

\begin{tabular}{lc}
\hline Variable & Mean or \% \\
\hline Age, years & $61 \pm 11$ \\
Female, \% & 29.6 \\
Previous myocardial infarction, \% & 38.0 \\
Revascularization, \% & 31.0 \\
Rest wall motion abnormalities & 40.9 \\
$\quad$ (RWMA), \% & $78 \pm 12$ \\
Body weight, kg & $87 \pm 11$ \\
Resting diastolic blood pressure, mmHg & $78 \pm 12$ \\
Peak diastolic blood pressure, mmHg & $152 \pm 26$ \\
Resting systolic blood pressure, mmHg & $151 \pm 31$ \\
Peak systolic blood pressure, mmHg & $79 \pm 15$ \\
Resting heart rate, bpm & $119 \pm 21$ \\
Peak heart rate, bpm & $12,030 \pm 3,545$ \\
Rate-pressure product ${ }^{\mathrm{a}}$ at rest & $17,935 \pm 4,807$ \\
Rate-pressure product at peak stress & $1.18 \pm 0,32$ \\
Wall Motion Score Index (WMSI) at & \\
$\quad$ baseline & $1.21 \pm 0.34$ \\
Wall Motion Score Index (WMSI) at peak & \\
$\quad$ dose &
\end{tabular}

Values are expressed as mean \pm SD or percentage

${ }^{\mathrm{a}}$ Rate-pressure product $=($ heart rate $) \times($ systolic blood pressure)

RWMA = Rest Wall Motion Abnormality; WMSI = Wall Motion Score Index

Dobutamine stress CMR with myocardial perfusion on peak-dose dobutamine

Eighteen of the 115 patients (16\%) had NWMA of whom 14 (78\%) showed perfusion deficits on peak-dose dobutamine in the corresponding segments. Four patients (22\%) with NWMA did not have a perfusion deficit. In these four patients, NWMA were attributable to a LBBB as could be confirmed with an independent (stress) ECG. Two of these were inducible LBBB, not known prior to the examination. CAG was positive for the corresponding segments in the 14 patients $(100 \%)$ with NWMA and a corresponding perfusion deficit.

Ninety-seven patients (84\%) had no NWMA. The perfusion images on peak-dose dobutamine showed absence of perfusion deficits in 67 of these 97 patients $(69 \%)$ and perfusion deficts in 30 $(31 \%)$. Of these patients $29(97 \%)$ were attributable to a known previous myocardial infarction in the patients history combined with the presence of RWMA. One patient (3\%) had a small perfusion deficit inferior which could not be assigned to a known previous event in the patients history. Figure 1 illustrates the course and outcome of the study. In Fig. 2 the additional value of cine or grid tagging images combined with stress first-pass perfusion images is illustrated.

Follow-up results (median 13.5 months, range 6-20 months) were obtained from all patients with NWMA's and a normal first-pass perfusion. None of these patients had cardiac events or revascularizations at follow-up.
Fig. 1 Flow chart illustrating course of the study and outcome NWMA = New Wall Motion Abnormality; LBBB = Left Bundle Branch Block; $\mathrm{CAG}=$ Coronary Angiogram

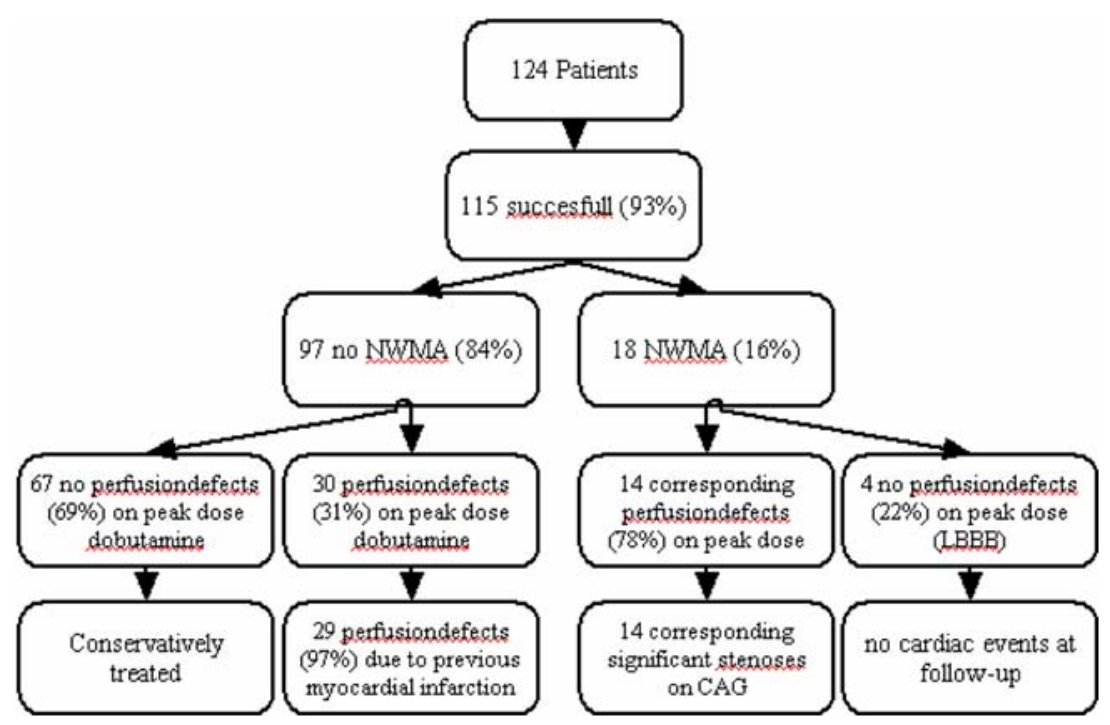


Fig. 2 Short axis views at peak-dose dobutamine. Cine image illustrating a NWMA inferior. (A) Perfusion abnormality in the corresponding segment. (B) Dyskinetic septal wall in another patient. (C) No perfusion abnormalities in the corresponding segment. (D) Dyskinetic septal wall in $\mathrm{C}$ was due to a $\mathrm{LBBB}$, this differentiation could be made by a perfusion sequence on peak-dose dobutamine. Arrows indicate the wall motion abnormality or perfusion abnormality NWMA = New Wall Motion Abnormality; LBBB = Left Bundle Branch Block
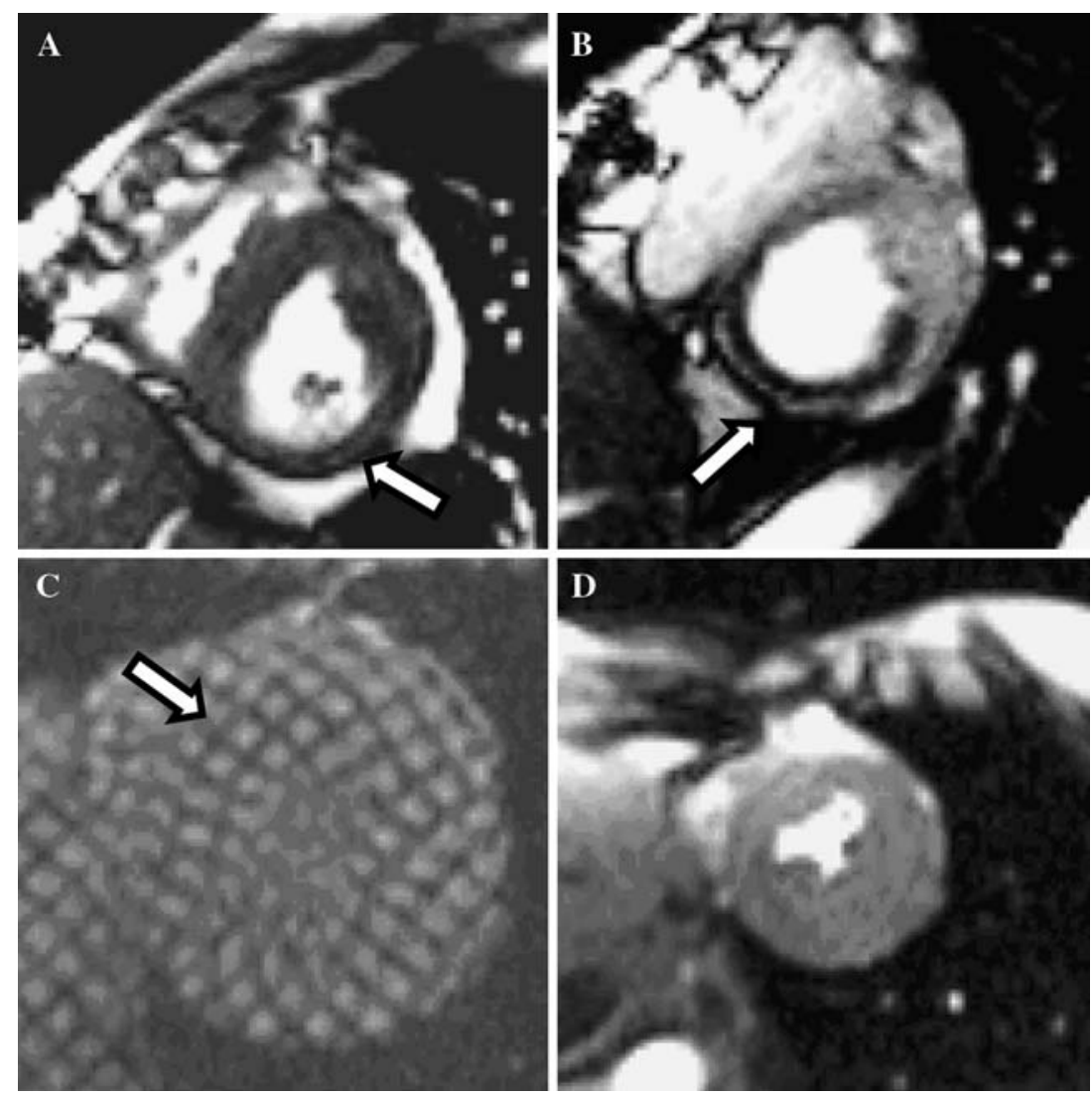

\section{Discussion}

This study demonstrates that adding first-pass myocardial perfusion imaging during peak-dose dobutamine has direct clinical relevance for the interpretation of dobutamine stress CMR examinations, by increasing the interpretation of possible wall motion abnormalities (NWMA) of the left ventricle. The addition of first pass perfusion relies on the conceptual use of the ischemic cascade. Ischemic wall motion abnormalities are preceded by perfusion abnormalities, therefore visualized NWMA due to myocardial ischemia should also show perfusion abnormalities. A normal perfusion study is used to identify wall motion abnormalities not due to myocardial ischemia, and an abnormal corresponding perfusion deficit is used to confirm NWMA, indicative for myocardial ischemia.

This is the first study to assess the additional value of a first pass myocardial perfusion imaging sequence on peak-dose dobutamine during a dobutamine stress CMR. Previous studies have reported a broad range of sensitivity and specificity for dobutamine stress CMR in the detection of myocardial ischemia [4]. In this protocol we have chosen for a prolonged infusion time of dobutamine from 3 to 6 min without atropine and the use of the target heart rate rule, as described before [4, 12-17].

The use of the target heart rate rule from a physical exercise based concept can not be generalized to a pharmacological stress setting and has been questioned in several reports [12-14, 18]. It has also been shown that the target heart rate rule can not be extrapolated to a pharmacological stress examination [19]. In a recent overview of published dobutamine stress CMR examinations by Strach et al. [20], the approach we used [4] showed to provide the highest diagnostic accuracy for significant coronary artery disease defined by a $>50 \%$ luminal stenosis on a coronary angiogram. Specificity, although already high, was lowered, according to 
our opinion, in part due to LBBB. This could be overcome with a perfusion sequence, taking into account the high negative predictive value of normal myocardial perfusion imaging. The additional value of myocardial perfusion MRI could be used for this purpose and may add significant diagnostic information.

In this study we added a perfusion sequence on peak-dose dobutamine in all patients. The results show a good agreement between the absence of NWMA and myocardial perfusion. The main purpose of this study was to assess the additional value of first-pass perfusion imaging in the presence of NWMA. Our recommendation for future clinical use is to add a first pass perfusion sequence on indication, namely if there is doubt on whether a NWMA is due to myocardial ischemia. In this way, the specificity of dobutamine stress CMR can be even further increased. By combining dobutamine stress CMR with myocardial perfusion, one still has the opportunity to examine for viability, which seems even superior to scar quantification [21].

Absolute specificity values cannot be given with this study, because a CAG was not performed in case of a negative dobutamine stress examination. The outcome of the dobutamine stress CMR and myocardial perfusion on peakdose dobutamine was used as a direct arbiter for subsequent clinical follow-up. In this respect follow-up was considered the reference standard. None of the patients with NWMA's and a normal myocardial perfusion had an adverse outcome at follow-up. Therefore, it is reasonable to state that adding first pass myocardial perfusion imaging on peak-dose dobutamine increases the specificity of dobutamine stress CMR in this patient group.

Our data could have been influenced by a referral bias, but in light of previous results, we found it not justifiable to perform a CAG in case of a negative examination [12]. Furthermore, the high negative predictive value of dobutamine stress CMR examination without perfusion imaging has been proven before [12, 22]. Mahrholdt et al. [23]. examined 139 patients with a LBBB. All 139 patients had fixed perfusion defects with SPECT, 19 could not be confirmed with CAG. Rest wall motion analysis and myocardial perfusion (between 5 and 30 min after contrast admin- istration) was performed on these 19 patients using MRI. All 19 patients showed septal wall motion abnormalities, but none showed subendocardial or transmural contrast enhancement. This also indicates that contrast enhanced imaging can help differentiate between WMA on the basis of coronary artery disease or a LBBB.

Long term follow-up will provide information about the case in which a small perfusion abnormality was seen inferior without NWMA. Whether this abnormality is a "true" abnormality or an artefact is unclear. This patient did not have any adverse cardiac event at 14 months follow-up.

Another limitation of our study is the fact that images were analysed semi-quantitatively. Quantitative wall motion analysis could possibly provide additional information, but due to the timeconsuming nature this is not yet feasible in clinical practice. Furthermore, no decisions can be made during the examination, which we believe is crucial, since overstressing may lead to serious complications [13].

Visual analysis of wall motion and perfusion images was performed in a consensus reading by an experienced radiologist and cardiologist. This can be regarded as a limitation since the observers are not blinded for the previous wall motion images. However, this does represent routine clinical practice and is fundamental to the underlying concept of using first-pass perfusion imaging in the presence of NWMA. In recent studies the inter-observer agreement of dobutamine stress CMR was investigated and good agreement was found $[24,25]$.

In a clinical post-infarct setting, delayed contrast enhancement imaging may provide valuable diagnostic information. To our opinion, this would in our study not have provided additional information regarding the fact whether a NWMA was caused by myocardial ischemia or a nonischemic cause, and guide the necessity for an invasive coronary angiogram. Cine and grid tagging images were acquired at rest to look for rest wall motion abnormalities. New wall motion abnormalities in this setting would represent myocardial ischemia (whether or not in the presence of a previous myocardial infarction). Delayed contrast enhancement imaging was therefore not performed in this protocol. 
In this protocol we only acquired stress firstpass perfusion images. Perfusion imaging at rest may be very useful in stress-rest perfusion imaging studies, but the main focus in this protocol is on a normal perfusion in case of NWMA. A rest perfusion exam would in this context not provide additional information (the rest perfusion exam will also be normal). In this way the protocol is not unnecessarily prolonged and a second bolus injection of gadolinium-DTPA can be omitted.

\section{Conclusion}

Good agreement exists between the absence of NWMA and a normal myocardial perfusion. Furthermore, a perfusion sequence on peak-dose dobutamine can help decide whether a NWMA is caused by ischemia or is due to a LBBB. First pass myocardial perfusion during peak-dose dobutamine can be used as an additional tool to reduce the number of false-positive NWMA's, to improve the detection of myocardial ischemia.

Acknowledgements The authors thank Stella Noach for critically reviewing the paper. Hester van der ZaagLoonen for statistical advice and Bernadette Blom for image acquisition.

\section{References}

1. Pennell DJ, Underwood SR, Manzara CC et al (1992) Magnetic resonance imaging during dobutamine stress in coronary artery disease. Am J Cardiol 70(1):34-40

2. Nagel E, Lehmkuhl HB, Bocksch W et al (1999) Noninvasive diagnosis of ischemia-induced wall motion abnormalities with the use of high-dose dobutamine stress MRI: comparison with dobutamine stress echocardiography. Circulation 99(6):763-770

3. Hundley WG, Hamilton CA, Thomas MS et al (1999) Utility of fast cine magnetic resonance imaging and display for the detection of myocardial ischemia in patients not well suited for second harmonic stress echocardiography. Circulation 100(16):1697-1702

4. Kuijpers D, Ho KY, van Dijkman PR et al (2003) Dobutamine cardiovascular magnetic resonance for the detection of myocardial ischemia with the use of myocardial tagging. Circulation 107(12):1592-1597

5. van Rugge FP, van der Wall EE, Spanjersberg SJ et al (1994) Magnetic resonance imaging during dobutamine stress for detection and localization of coronary artery disease. Quantitative wall motion analysis using a modification of the centerline method. Circulation 90(1):127-138

6. Nesto RW, Kowalchuk GJ (1987) The ischemic cascade: temporal sequence of hemodynamic, electrocardiographic and symptomatic expressions of ischemia. Am J Cardiol 59(7):23C-30C

7. Schinkel AF, Elhendy A, Bax JJ et al (2006) Prognostic implications of a normal stress technetium-99 m-tetrofosmin myocardial perfusion study in patients with a healed myocardial infarct and/or previous coronary revascularization. Am J Cardiol 97(1):1-6

8. Elhendy A, Schinkel AF, van Domburg RT et al (2005) Risk Stratification of Patients with Angina Pectoris by Stress $99 \mathrm{mTc}$-Tetrofosmin Myocardial Perfusion Imaging. J Nucl Med 46(12):2003-2008

9. Schinkel AF, Elhendy A, van Domburg RT et al (2003) Incremental value of exercise technetium-99 m tetrofosmin myocardial perfusion single-photon emission computed tomography for the prediction of cardiac events. Am J Cardiol 91(4):408-411

10. Hachamovitch R, Hayes S, Friedman JD et al (2003) Determinants of risk and its temporal variation in patients with normal stress myocardial perfusion scans: what is the warranty period of a normal scan? $\mathrm{J}$ Am Coll Cardiol 41(8):1329-1340

11. Wintersperger BJ, Penzkofer HV, Knez A et al (1999) Multislice MR perfusion imaging and regional myocardial function analysis: complimentary findings in chronic myocardial ischemia. Int $\mathrm{J}$ Card Imaging 15(6):425-434

12. Kuijpers D, van Dijkman PR, Janssen CH et al (2004) Dobutamine stress MRI. Part II. Risk stratification with dobutamine cardiovascular magnetic resonance in patients suspected of myocardial ischemia. Eur Radiol 14(11):2046-2052

13. Kuijpers D, Janssen CH, van Dijkman PR, Oudkerk M (2004) Dobutamine stress MRI. Part I. Safety and feasibility of dobutamine cardiovascular magnetic resonance in patients suspected of myocardial ischemia. Eur Radiol 14(10):1823-1828

14. Kuijpers D (2005) Diagnosis of coronary artery disease with dobutamine-stress MRI. Eur Radiol 15(Suppl 2): B48-B51

15. Janssen $\mathrm{CH}$, Kuijpers D, Vliegenthart R, Overbosch J, van Dijkman PR, Zijlstra F, Oudkerk M (2005) Coronary artery calcification score by multislice computed tomography predicts the outcome of dobutamine cardiovascular magnetic resonance imaging. Eur Radiol 15(6):1128-1134

16. van Dijkman PR, Kuijpers DA, Blom BM, van Herpen G (2002) Dobutamine stress magnetic resonance imaging: a valuable method in the noninvasive diagnosis of ischemic heart disease. J Electrocardiol 35(Suppl):57-59

17. van Dijkman PR, Kuijpers TJ, Blom BM, van Herpen $G$ (2002) [Dobutamine stress magnetic resonance imaging (DS-MRI), a valuable tool for the diagnosis of ischemic heart disease]. Ned Tijdschr Geneeskd 146(28):1327-32 
18. Elhendy A, van Domburg RT, Bax JJ, Nierop PR, Geleijnse ML, Ibrahim MM, Roelandt JR (1999) The functional significance of chronotropic incompetence during dobutamine stress test. Heart 81(4):398-403

19. Arsenault M, Bergeron S, Dumesnil JG, Fortin MP, Poirier P (2005) Anginal hreshold between stress tests: exercise versus dobutamine stress echocardiography. Med Sci Sports Exerc 37(1):18-23

20. Strach K, Meyer C, Schild H, Sommer T (2006) Cardiac stress MR imaging with dobutamine. Eur Radiol 16(12):2728-2738

21. Wellnhofer E, Olariu A, Klein C et al (2004) Magnetic resonance low-dose dobutamine test is superior to SCAR quantification for the prediction of functional recovery. Circulation 109(18):2172-2174

22. Hundley WG, Morgen TM, Neagle CM et al (2002) Magnetic resonance imaging determination of cardiac prognosis. Circulation 106:2328-2333
23. Mahrholdt H, Zhydkov A, Hager S et al (2005) Left ventricular wall motion abnormalities as well as reduced wall thickness can cause false positive results of routine SPECT perfusion imaging for detection of myocardial infarction. Eur Heart J 26(20):2127-2135

24. Syed MA, Paterson DI, Ingkanisorn WP, Rhoads KL, Hill J, Cannon RO III, Arai AE (2005) Reproducibility and inter-observer variability of dobutamine stress CMR in patients with severe coronary disease: implications for clinical research. J Cardiovasc Magn Reson 7(5):763-768

25. Paetsch I, Jahnke C, Ferrari VA, Rademakers FE, Pellikka PA, Hundley WG, Poldermans D, Bax JJ, Wegscheider K, Fleck E, Nagel E (2006) Determination of interobserver variability for identifying inducible left ventricular wall motion abnormalities during dobutamine stress magnetic resonance imaging. Eur Heart J 27(12):1459-1464 